\title{
Optimal Pricing Based on Real-time Monitoring of Hotel Information
}

\author{
Xiaojie Chen, ${ }^{1}$ Chan Wang, ${ }^{2 *}$ Jiali Chen, ${ }^{1}$ \\ Huixu Yang, ${ }^{2 * *}$ Chih-Hsien Hsia, ${ }^{3}$ and Shun-Jhen Huang ${ }^{4}$ \\ ${ }^{1}$ College of Mathematics and Information Engineering, Longyan University, Fujian 364012, China \\ ${ }^{2}$ School of Humanities, Jinan University, Guangdong 519070, China \\ ${ }^{3}$ Department of Computer Science and Information Engineering, National Ilan University, Yilan 260, Taiwan \\ ${ }^{4}$ Graduate Institute of Digital Mechatronic Technology, Chinese Culture University, Taipei 11114, Taiwan
}

(Received December 31, 2020; accepted May 24, 2021)

Keywords: monitoring system, crawler program, best pricing, data mining, software testing

Online travel agencies (OTAs) are becoming popular nowadays. Travelers now search for and compare room prices of hotels online, which makes the competition between hotels in terms of room price fierce. For convenient and precise information on room prices, we propose a realtime monitoring system of room prices for hotel management to follow the changes in hotel information. The system finds the best price and provides a reference room price. The results show that the proposed method proposes the optimal price by considering competitors' prices. The results of this research provide a basis for improving existing travel and hotel reservation services for travelers.

\section{Introduction}

The rapid development of the Internet has led to the appearance of online travel agency (OTA) platforms that enable tourists to make room reservations conveniently. The transparent information on the platforms enables users to compare rates and choose their accommodation. $^{(1-6)}$ Recently, hotels have adopted IoT technology to benefit hotel operators and guests. As equipped with IoT devices that use various sensors, hotels provide voice control in rooms, digital concierge, contactless payment methods, and contact tracing and crowd control. Information collected from the devices and sensors are directed to OTAs to increase sales efficiently in a competitive industry. Hotels are cooperating with OTAs to provide accommodation services for attracting guests. ${ }^{(7)}$

The hotel industry of Taiwan has observed an increase in the number of tourists. However, the increasing number of hotels has led to declining occupancy rates of hotels, increasing the ferocity of the competition despite the popularity of OTAs. Thus, operators and managers of hotels seek an appropriate pricing policy that maximizes the benefits in a sales period. Anjos et $a l .{ }^{(8)}$ and Gallego and Ryzin ${ }^{(9)}$ used intensity control theory to propose an optimal price for the time expiration and product rate with different demand functions. Koide and Ishii proposed ${ }^{(10)} \mathrm{a}$

\footnotetext{
*Corresponding authors: e-mail: mschanw@163.com

** Corresponding authors: e-mail: yanghuixu@jnu.edu.cn https://doi.org/10.18494/SAM.2021.3278
} 
policy of optimal room allocation for early-bird discounts, overbooking, and regular prices. Chen and Kachani proposed a room allocation policy to maximize hotel revenue by imposing different reservation restrictions on customers in accordance with their previous fulfillment or cancellation, in addition to a prediction module for optimizing room allocation. ${ }^{(11)}$ Aziz et al. ${ }^{(12)}$ proposed a dynamic pricing model that allocated room capacity to different customer groups by accommodation type to maximize hotel revenue. They did not apply rooms sold by different sales methods as decision variables, as in the traditional model of revenue management, but regarded the prices of different segments as decision variables to increase hotel revenue. A dynamic rate model that used the concept of the four multipliers, time, capacity, stay, and occupancy, was proposed by Zhang et al., where the appropriate rate was calculated with various parameters. ${ }^{(13)}$ They considered the impact of price on demand but did not provide an appropriate basis for the price elasticity in detail.

In previous studies, the influence of competitors' prices and the selling price were not considered in the optimization of the price of rooms. Therefore, we propose a management and monitoring system that monitors the price changes of competitors and immediately informs the hotel operators/managers of the changes. This basic information helps to respond to competitors' price changes and thus increase a hotel's revenue.

This paper is organized as follows. In Sect. 2, we describe the process by which the system monitors hotel information. In Sect. 3, the results of data analysis and experiments are shown, and Sect. 4 is the conclusion of the paper.

\section{System for Monitoring Hotel Information}

\subsection{System architecture diagram}

The proposed system follows a four-step process: data collection, data analysis, information visualization, and notification. The overall system architecture is shown in Fig. 1.

\subsection{Subsystem description}

\subsubsection{Data collection}

Bezzubtsevav and Ignatov ${ }^{(14)}$ used a crawler program to collect data from a data source (www.booking.com) and explored whether it was beneficial to book a room in advance. Here, we applied the crawler program for OTA data collection. According to SimilarWeb, booking.com was ranked first in the category of hotel accommodation searches, which indicated the credibility of the data. Therefore, we designed a data crawler program for booking.com that simulates users' web browsing behavior and interaction, processes the HTML tags on the website page, and finds regular expressions of strings to obtain the required information.

The data crawler program has the following three processing steps.

Step 1: The page data of the webpage is presented. The page data is divided into front- and backend separations to generate web pages. The back-end data processing program directly processes 


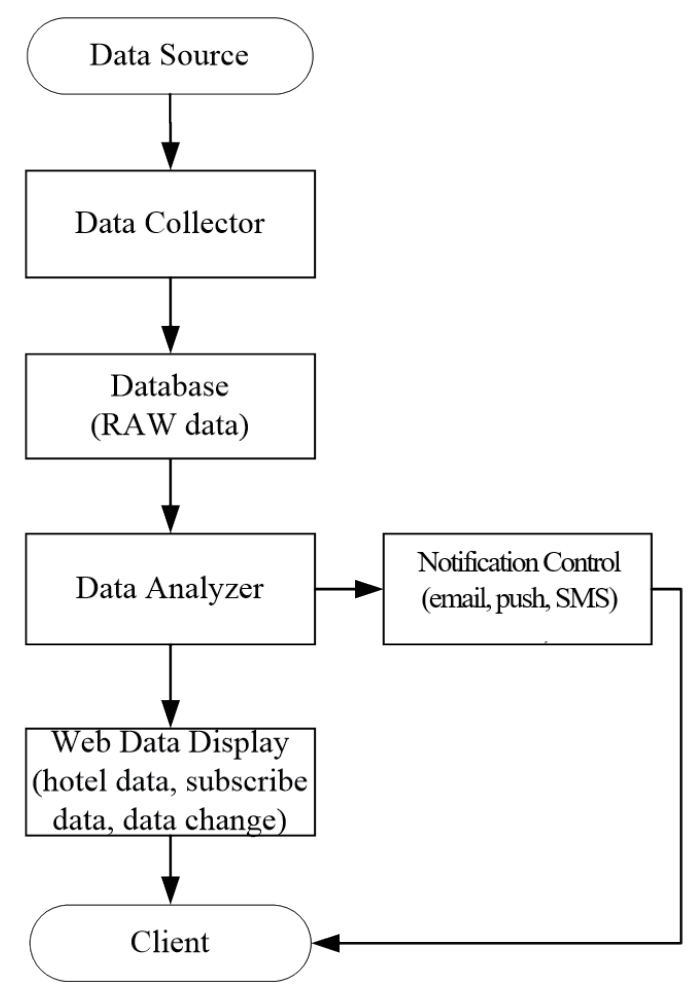

Fig. 1. System architecture of monitoring hotel information.

the data and outputs the website pages. The webpage generation is based on the data processed by the back-end program and transmitted to the front-end program through the application programming interface (API) for page rendering. The crawler finds the API path, compares and analyzes the API data, and then stores it in the database. The pages generated by the back-end processing program are collated to generate a page presenting the results. Therefore, the program analyzes the page with HTML tags and data regulation to obtain the data required for the analysis. As the booking.com website is a back-end page-generation site, it is necessary to analyze the HTML tags on the website and the parameters on the address bar. The information on the website includes information of hotels such as the check-in date, project room types, project rate, project reservation instructions, and remaining rooms. A real case of a hotel in Taipei City is used as an example in Table 1.

Step 2: The crawler flowchart is shown in Fig. 2 and is described as follows. After analyzing the information on the website, the log-in, check-in, and dates are crawled from the database to find guests who have booked in the previous 30 to 45 days. Therefore, the reservation webpages of the previous 30 days are searched and saved to the local server. Considering the crawling time limit and the response speed of the website page, the query is executed every 30 min to gather changes in the hotel information.

Step 3: Changes in the website maintenance page and the crawling data are detected by using the crawler program. 
Table 1

Content analysis of hotel information of a hotel in Taipei City.

\begin{tabular}{|c|c|c|}
\hline Items & Program command & Result \\
\hline $\begin{array}{l}\text { Number of people } \\
\text { suitable for the project }\end{array}$ & $\begin{array}{l}\text { \$('tr[data-block-id="55587203_105643439_0_2_0”]. } \\
\text { hprt-table-cell-occupancy.invisible_spoken').text(); }\end{array}$ & Maximum number of people: 2 \\
\hline Room type & $\begin{array}{l}\text { \$('tr[data-block-id="55587203_105643439_0_2_0”]. } \\
\text { hprt-roomtype-icon-link').text(); }\end{array}$ & $\begin{array}{l}\text { Room with king-size bed } \\
\text { and balcony }\end{array}$ \\
\hline Room price & $\begin{array}{l}\text { \$('tr[data-block-id="55587203_105643439_0_2_0”]. } \\
\text { hprt-price-price span').text(); }\end{array}$ & NT\$ 6544 \\
\hline $\begin{array}{l}\text { Number of remaining } \\
\text { rooms }\end{array}$ & $\begin{array}{l}\begin{array}{l}\$(\text { tr[data-block-id="55587203_105643439_0_2_0"]. } \\
\text { hprt-nos-select').length; }\end{array} \\
\text {. }\end{array}$ & 1 \\
\hline Booking notice & $\begin{array}{c}\$(\text { 'tr[data-block-id="55587203_105643439_0_2_0”] } \\
\text { hprt-table-cell-conditions span').text(); }\end{array}$ & Discounted, non-refundable \\
\hline
\end{tabular}
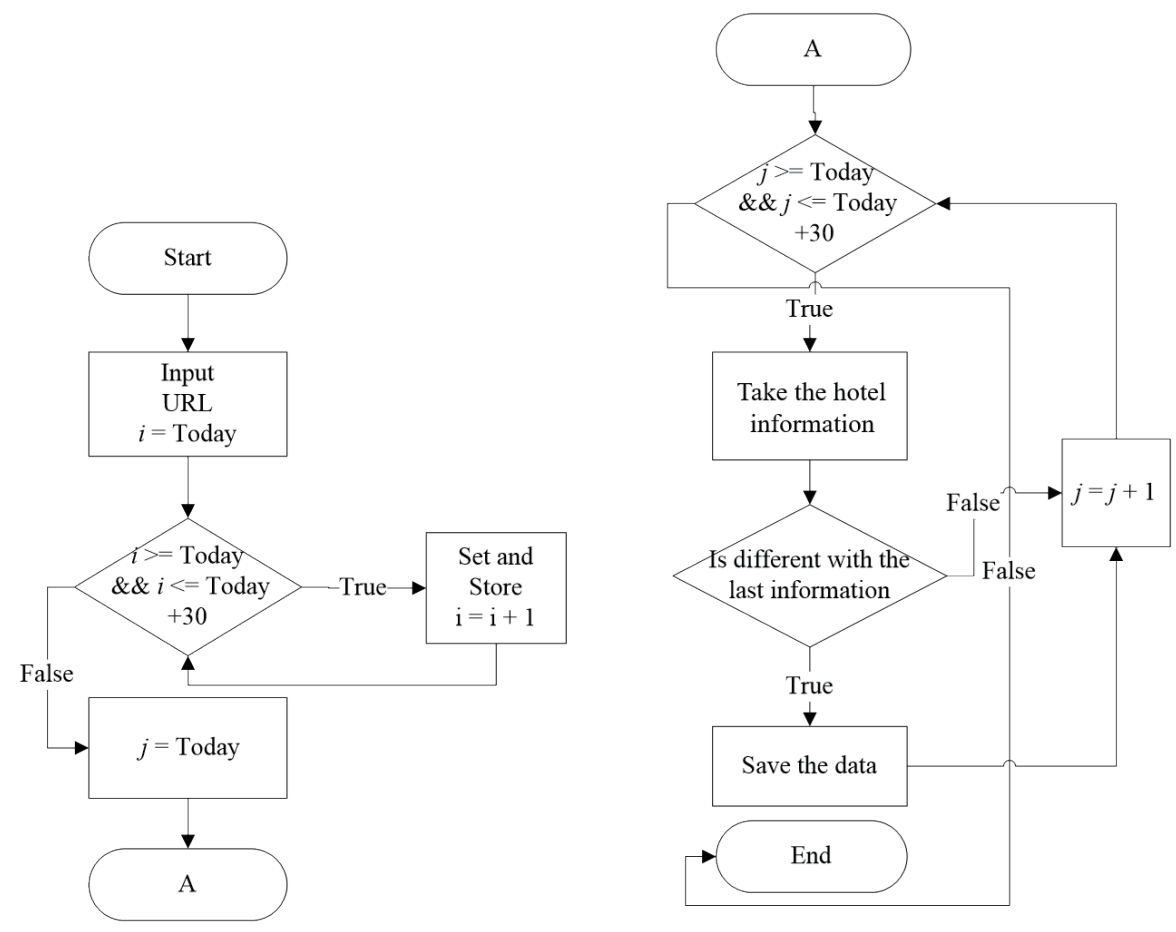

Fig. 2. Design process of crawling.

\subsubsection{Data analysis}

After querying the database, hotel information such as the type and number of rooms, stay period, and rate is integrated. Then, data can be compared to obtain the total number of rooms and sold rooms, rate changes, and sales revenue.

The crawled data in the local database are compared with the website page information every $30 \mathrm{~min}$, similarly to in the first step of the analysis. If there are any changes, the rate and number of remaining rooms are stored in the rate and stock $\log$ form of the database. The form includes the changes in the data, check-in date, and crawling time for information visualization and change notification. 


\subsubsection{Information visualization}

The results of the data analysis are shown on a website, making it convenient for hotel operators/managers to see the trends and sales of competitors' hotel rooms. As shown in Figs. 3-5, an information website presents the collated data as charts so that the hotel

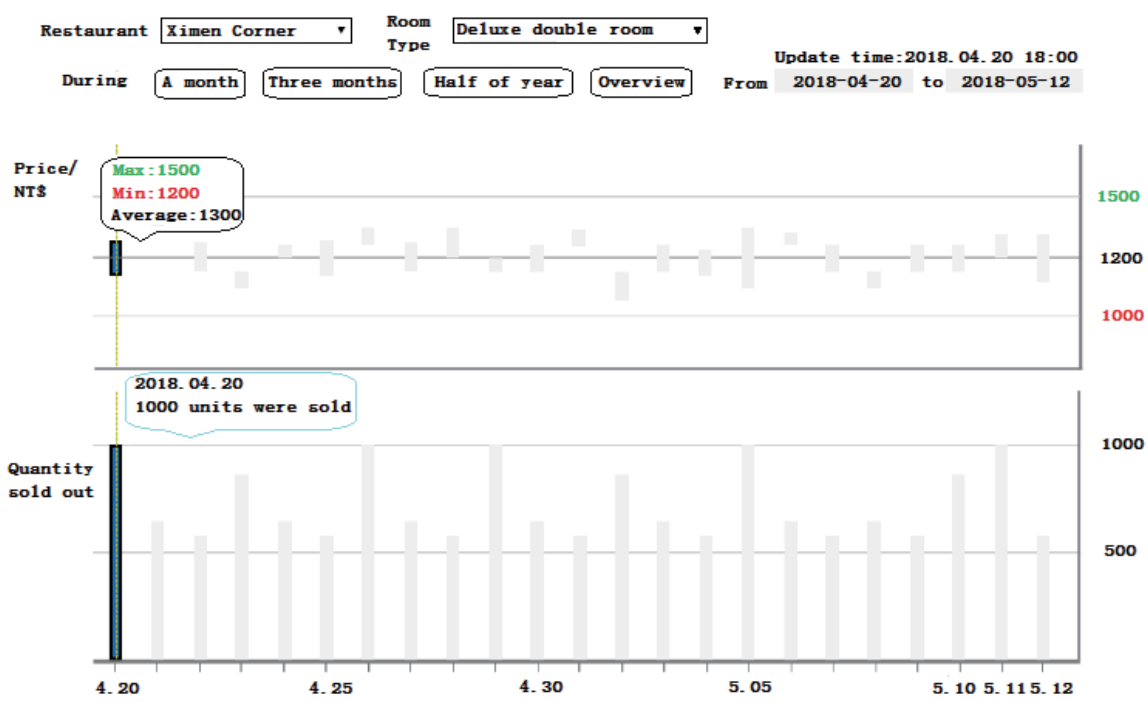

Fig. 3. (Color online) Room monitoring report.

\begin{tabular}{|c|c|c|c|c|c|c|}
\hline $\begin{array}{c}\text { Change } \\
\text { time }\end{array}$ & $\begin{array}{c}\text { Check in } \\
\text { time }\end{array}$ & restaurant & $\begin{array}{c}\text { Room } \\
\text { Type }\end{array}$ & $\begin{array}{c}\text { Price } \\
\text { before } \\
\text { change }\end{array}$ & $\begin{array}{c}\text { Price } \\
\text { after } \\
\text { change }\end{array}$ & Percentage \\
\hline $\begin{array}{c}2018 / 01 / 12 \\
11: 30 \mathrm{pm}\end{array}$ & $2018 / 01 / 29$ & As & $\begin{array}{c}\text { Double } \\
\text { room }\end{array}$ & 1800 & 1900 & $106 \%$ \\
\hline $\begin{array}{c}2018 / 01 / 12 \\
11: 30 \mathrm{pm}\end{array}$ & $2018 / 01 / 29$ & As & $\begin{array}{c}\text { Double } \\
\text { room }\end{array}$ & 1800 & 1900 & $106 \%$ \\
\hline
\end{tabular}

Fig. 4. List of changes in hotel rate.

January

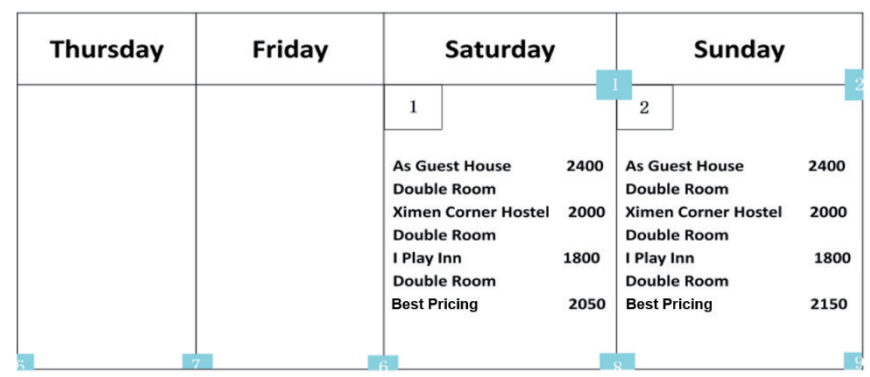

Fig. 5. (Color online) Monitoring calendar. 
operator/manager can easily browse the information of competitors' hotels that has changed. The information on the website displays monitoring reports, the list of changed rates and rooms, and competitors' occupancy rates. A calendar of monitoring reports is provided for the convenience of hotel operators/managers.

\subsubsection{Change notification}

The changes in competitors' information on room prices and the number of rooms are notified immediately through a function on the website. Operators/managers can choose the notification conditions as required. If users do not adjust the rate and the number of rooms, no changes will be delivered through the command of 'email.room price' The operation process is shown in Fig. 6.

\section{Analysis and Results}

Hotel information includes non-storability of rooms, high fixed costs, and demand volatility. Non-storability means that rooms are only valid until they are reserved. After the reservation, the rooms are not available in the future. Fixed costs do not change with the company's products and sales under certain operating conditions. Demand volatility means that the current demand cannot be preset. Customers often cancel reservations due to weather or personal factors. ${ }^{(15)}$ The demand forecast in this system uses real-time data to make changes and reduce forecasting errors. Koide and Ishii used historical data to perform a regression analysis to derive the required parameters and to optimize the demand of rooms with the room prices and costs by using ${ }^{(10)}$

$$
Q=\frac{1}{2}\left(\left(1-\frac{1}{\beta}\right) r_{0}+v\right),
$$

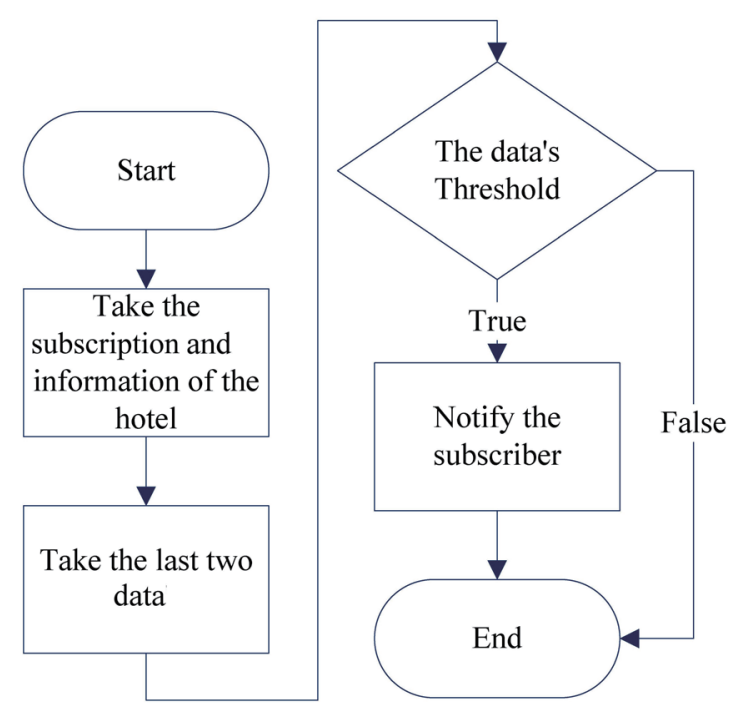

Fig. 6. Subscription notification process. 
where $Q$ is the demand (quantity) of rooms, $\beta$ denotes the price elasticity of demand, $r_{0}$ is the base price, and $v$ is the cost.

The real-time hotel information is crawled back to obtain the best selling prices using the required parameters to maximize the hotel's profit. The value of $\beta$ is obtained by applying the changes in the prices and the number of remaining rooms in real time. The price elasticity of demand is defined as the change in demand in accordance with the price. The demand function of individual competitors to obtain $\beta_{n}$ is

$$
\beta_{n}=\frac{-\Delta H_{n} r}{-\Delta H_{n} p}
$$

Equations (3) and (4) are the demand function of each competitor with the weight parameters $\left(\alpha_{n}\right)$ and $\beta_{t}$ and the basic price with the weight parameters, respectively:

$$
\begin{aligned}
& \beta_{T}=\frac{\beta_{1} * \alpha_{1}+\beta_{2} * \alpha_{2}+\cdots+\beta_{n} * \alpha_{n}}{\alpha_{1}+\alpha_{2}+\cdots+\alpha_{n}}=\frac{\sum_{k=1}^{n}\left(\beta_{n} * \alpha_{n}\right)}{\sum_{k=1}^{n} \alpha_{n}}, \text { and } \\
& r_{0}=\frac{H_{1} r * \alpha_{1}+H_{2} r * \alpha_{2}+\cdots+H_{n} r * \alpha_{n}}{\alpha_{1}+\alpha_{2}+\cdots+\alpha_{n}}=\frac{\sum_{k=1}^{n} H_{n} r * \alpha_{n}}{\sum_{k=1}^{n} \alpha_{n}},
\end{aligned}
$$

where $\beta_{n}$ is the price elasticity of demand for each hotel, $\beta_{T}$ is the price elasticity of demand after weight processing, $H_{n} r$ is the number of remaining rooms in the hotel, $H_{n} p$ is the hotel room price, $\alpha$ is the weight parameter $(0<\alpha<10), H_{n} r_{x}$ is the current number of remaining rooms, and $H_{n} p_{x}$ is the current hotel room price.

Koide and Ishii ${ }^{(10)}$ combined Eqs. (1), (3), and (4) to derive the following optimal price:

$$
1-\beta\left(\sum_{k=1}^{n}\left(\frac{-\left(H_{n} r_{x}-H_{n} r_{x-1}\right)}{H_{n} p_{x}-H_{n} p_{x-1}} * \frac{H_{n} p_{x}}{H_{n} r_{x}} * \alpha_{n}\right) / \sum_{k=1}^{n} \alpha_{n}\right)=\sum_{k=1}^{n}\left(H_{n} r * \alpha_{n}\right) / \sum_{k=1}^{n} \alpha_{n}+v,
$$

where $v$ is the variable cost.

The data from three hotels with the same star rating and similar reviews were crawled and verified using the equations. The results are shown in Fig. 7. The trend of optimized prices is similar among the hotels. Since Eq. (5) is derived from the price elasticity of demand, the price is corrected when it does not meet the price elasticity of demand. For example, on February 25, two hotels lowered their room prices, while the third increased them. The best price calculated by the program of this study was the same as that of the two hotels. In this case, increasing the price is unreasonable. The best price was calculated using Eq. (5) in accordance with the current price elasticity of demand. In general, the calculated best prices were lower than the real prices of the hotels, which made the reservation of the rooms difficult. 


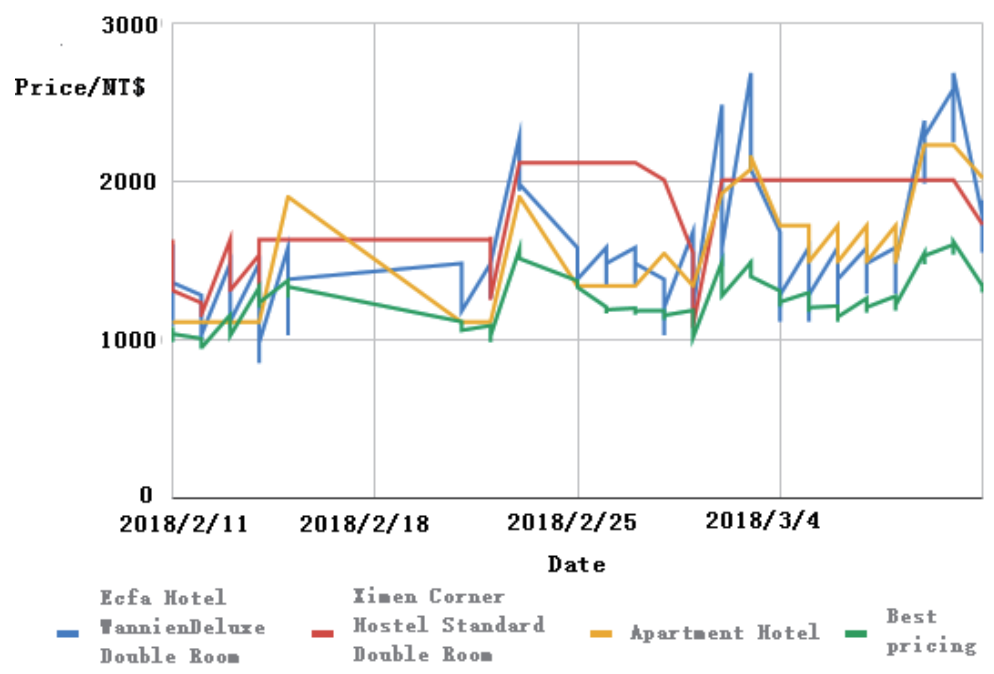

Fig. 7. (Color online) Analysis results of three hotels with the same star rating and similar comments.

\section{Conclusions}

A hotel monitoring and management system with real-time data was developed. The system calculates the parameters to obtain demand and optimal prices in real time. The room price is adjusted to increase the check-in rate and revenue and optimized by using the system. The result of this study provides a basis for future research on the effects of weather, flights, number of visitors from abroad, national holidays, Google trends, information on all hotels, and lowest prices. To observe such effects on room prices, the accuracy of the demand forecast needs improvement to derive a precise reference price.

\section{Acknowledgments}

This work was supported by the Young and Middle-aged Teacher Program of the Education Department of Fujian (JAT170579).

\section{References}

1 G. Viglia, A. Mauri, and M. Carricano: Int. J. Hospitality Manage. 52 (2016) 46. https://doi.org/10.1016/j. ijhm.2015.09.010

2 Y. Liu, X. Han, Z. Zhao, Y. Zhang, and J. Sun: Proc. 2019 Int. Conf. Artificial Intelligence and Advanced Manufacturing (AIAM, 2019) 223. https://doi.org/10.1109/AIAM48774.2019.00052

3 W. Li, P. Svärd, J. Tordsson, and E. Elmroth: Proc. 2013 IEEE/ACM 6th Int. Conf. Utility and Cloud Computing (2013) 187. https://doi.org/10.1109/CGC.2012.90

4 Y. Zhai and Q. Zhao: Proc. 2016 IEEE Int. Conf. Acoustics, Speech and Signal Processing (ICASSP, 2016) 4772. https://doi.org/10.1109/ICASSP.2016.7472583

5 H. Yeganeh, A. Salahi, and M. A. Pourmina: Can. J. Electr. Comput. Eng. 42 (2019) 41. https://doi.org/10.1109/ CJECE.2019.2890833

6 J. Huang, L. Zhu, B. Fan, Y. Chen, W. Jiang, and S. Li: IEEE Access 6 (2018) 69807. https://doi.org/10.1109/ ACCESS.2018.2879824 
7 L. Ling, X. Guo, and L. Liang: Proc. 2009 Int. Conf. Computational Intelligence and Software Engineering (2009) 1. https://doi.org/10.1109/CISE.2009.5362585

8 M. F. Anjos, R. C.-H. Cheng, and C. S. M. Currie: Eur. J. Oper. Res. 166 (2005) 246. https://doi.org/10.1016/j. ejor.2004.02.015

9 G. Gallego and G. V. Ryzin: Manage. Sci. 40 (1994) 999. https://doi.org/10.1287/mnsc.40.8.999

10 T. Koide and H. Ishii: Int. J. Prod. Econ. 93-94 (2005) 417. https://doi.org/10.1016/j.ijpe.2004.06.038

11 C. Chen and S. Kachani: J. Revenue Pricing Manage. 6 (2007) 163. https://doi.org/10.1057/palgrave. rpm.5160082

12 H. A. Aziz, M. Saleh, M. H. Rasmy, and H. ElShishiny: Egypt. Inf. J. 12 (2011) 177. https://doi.org/10.1016/j. eij.2011.08.001

13 Q. Zhang, L. Qiu, H. Wu, J. Wang, and H. Luo: Proc. 2019 Int. Conf. Data Mining Workshops (ICDMW, 2019) 370. https://doi.org/10.1109/ICDMW.2019.00061

14 A. Bezzubtseva and D. I. Ignatov: Proc. 2013 IEEE Int. Conf. Data Mining Workshops (2013) 426. https://doi. org/10.1109/ICDMW.2013.32

15 W. Wei and H. Lee: Proc. 2009 Int. Conf. Business Intelligence and Financial Engineering (2009) 853-856. https://doi.org/10.1109/bife.2009.195 